\title{
The Construction and Optimization of Purifying Performance Evaluation System of Non-woven Filter Materials
}

\author{
Minghu $\mathrm{An}^{1}$, Baoi Shi ${ }^{1,2}$, Guang Yang ${ }^{1}$,Wei Zhang ${ }^{1,2, a}$ \\ ${ }^{1}$ College of Textile and Garment, Hebei University of Science and Technology, Shijiazhuang, \\ Hebei, 050018, China \\ ${ }^{2}$ Research Center of Engineering and Technology of Textile and Garment, Hebei Province, \\ Shijiazhuang, Hebei, 050031, China \\ ${ }^{a}$ Corresponding author Email: zhwei69@126.com
}

Keywords: Purifying Performance, Air Filter Materials, Multiple Regression Analysis, Unified Objective Method.

Abstract. According to Chinese National Standards (GB/T14295-2008,GB/T6165-2008), European standard(EN779:2012) and American standards(ASHRAE52.1-1992, ASHRAE52.2-2007), a testing device of filter materials' performance was set up. Using the filtration efficiency and resistance of acupuncture, meltblown and electret non-woven filter materials gathered from laboratory, purifying performance mathematical models were established and optimized with multiple regression analysis. With unified objective function, a comprehensive evaluation system was established. The research can provide important reference to choose and use non-woven filter materials to purify fine particles in ambient air.

\section{Introduction}

Ambient Air Quality Standard (2012) published by NEPA claimed the annual and daily PM $_{2.5}$ concentration should be less than $0.035 \mathrm{mg} / \mathrm{m}^{3}$ and $0.075 \mathrm{mg} / \mathrm{m}^{3}$ in china[1].However, improving ambient air quality is a long-term project, so people pay more attention to the indoor particle concentration. Using filters with high efficiency and low resistance to purify fine particles is an effective way to solve the problem. As there is no national standard for filter materials in china, so construction and optimization of filter's purifying performance evaluation system becomes important to select and use filters.

\section{Trapping mechanism of filter materials}

With direct intercept, static, diffusion, sieve and inertia, filter materials separate particles from the air. While single fiber trapping particles, all factors are at work. However, each effect is not the same for different particles, and all effect has joint roles. The comprehensive filtration efficiency curves were shown in Fig.1.

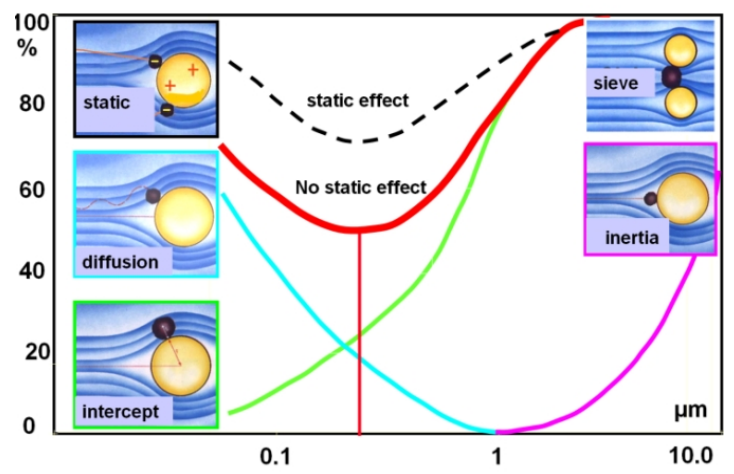

Fig.1 Trapping mechanisms 
Comprehensive filtration efficiency is not a simple superposition of different trapping effects. The filtration efficiency to larger particles is better than to smaller particles. Electrostatic interaction can improve filtration efficiency to the small particles obviously [2].

The fibers in non-woven filters can be split evenly and do not have a clear direction, with a large number of fluffy pore structures, the fibers can trap the particles in the pores with fluffy extensive contacts. Therefore, the filters have higher filtration efficiency and lower pressure loss. Based on the mechanical trapping mechanisms above, Electret filters with a large number of fixed charges make use of electrostatic effect to trap particulate matter, especially fine particulate matters. Meanwhile, the deposited particulate matters are more conductive than the air, they will make the electric field more concentrated, then the particles will be deposited in the former, which lead to higher filtering efficiency, lower resistance and make cleaning easier [3-4].

\section{Experiments}

According to Chinese National Standards (GB/T14295-2008, GB/T6165-2008), European standard (EN779:2012) and American standards (ASHRAE52.1-1992, ASHRAE52.2-2007), a testing device of filter materials' performance was set up as shown in Fig.2. With the device, the filters' mass concentration of particles, filtration efficiency and filtration resistance at different velocity were tested [5-8].

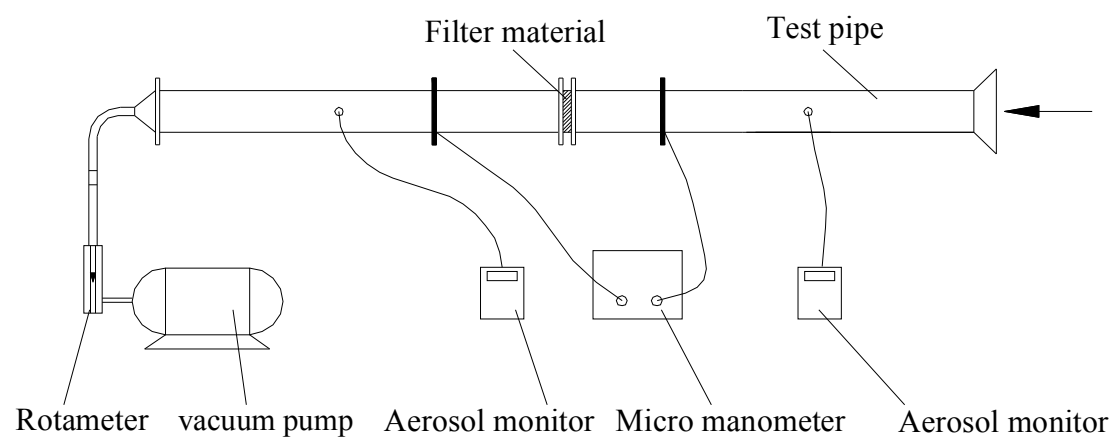

Fig. 2 Testing device

The purifying performance of different polypropylene filters for $\mathrm{PM}_{2.5}$ in the ambient air were tested under wind velocity of $0.2 \mathrm{~m} / \mathrm{s}$ and $0.5 \mathrm{~m} / \mathrm{s}$. The filters' specifications and purifying performance were shown in Tab.1.

Tab.1 Filters' specifications and purifying performance

\begin{tabular}{|c|c|c|c|c|c|c|}
\hline \multirow{2}{*}{ Code } & \multirow{2}{*}{$\begin{array}{c}\text { Areal density } \\
\quad / \mathrm{g} \cdot \mathrm{m}^{-2}\end{array}$} & \multirow{2}{*}{$\begin{array}{l}\text { Fiber diameter } \\
\qquad / \mu \mathrm{m}\end{array}$} & \multicolumn{2}{|c|}{ Filter efficiency/\% } & \multicolumn{2}{|c|}{ Filter resistance $/ \mathrm{Pa}$} \\
\hline & & & $0.2 \mathrm{~m} / \mathrm{s}$ & $0.5 \mathrm{~m} / \mathrm{s}$ & $0.2 \mathrm{~m} / \mathrm{s}$ & $0.5 \mathrm{~m} / \mathrm{s}$ \\
\hline $\mathrm{R}_{1}$ & 78 & 7.0 & 71.3 & 64.0 & 120 & 330 \\
\hline $\mathrm{R}_{2}$ & 152 & 7.0 & 81.2 & 76.0 & 150 & 400 \\
\hline$Z_{1}$ & 340 & 11.5 & 35.0 & 25.0 & 50 & 140 \\
\hline $\mathrm{Z}_{2}$ & 160 & 18.0 & 25.5 & 15.8 & 6 & 38 \\
\hline $\mathrm{J}_{1}$ & 130 & 21.5 & 92.5 & 85.3 & 13 & 41 \\
\hline $\mathrm{J}_{2}$ & 150 & 21.5 & 94.6 & 87.3 & 15 & 51 \\
\hline
\end{tabular}

Where, $R_{1}$ and $R_{2}$ are melt-blown filters, $Z_{1}$ and $Z_{2}$ are acupuncture filters, $J_{1}$ and $J_{2}$ are needle electret filters.

The data above can only illustrate the numerical value of filter efficiency and filter resistance, but they can't determine the influence of weight, fiber diameter and velocity to the purifying performance, and can't make a comprehensive evaluation on filtering performance. So the paper built purifying performance mathematical models with multiple regression analysis, and optimized the model with 
analysis of variance. With unified objective function, a comprehensive evaluation system was established.

\section{Construction and optimization of mathematical models}

Multiple Regression Analysis. The filtering performances are influenced by many factors and their interaction, they can't be described by an explicit mathematical expression. Therefore, it is an effective way to establish polynomial expression to approximate the relationship between single filtering performances index and its influence factors. Firstly, multiple regression analysis was made to the established polynomial model in a certain confidence level. If the equation is significant, the coefficient significance were tested, then insignificant coefficient were removed in order to create the mathematical model of single filtering performances, the steps are as shown in Figure 3.

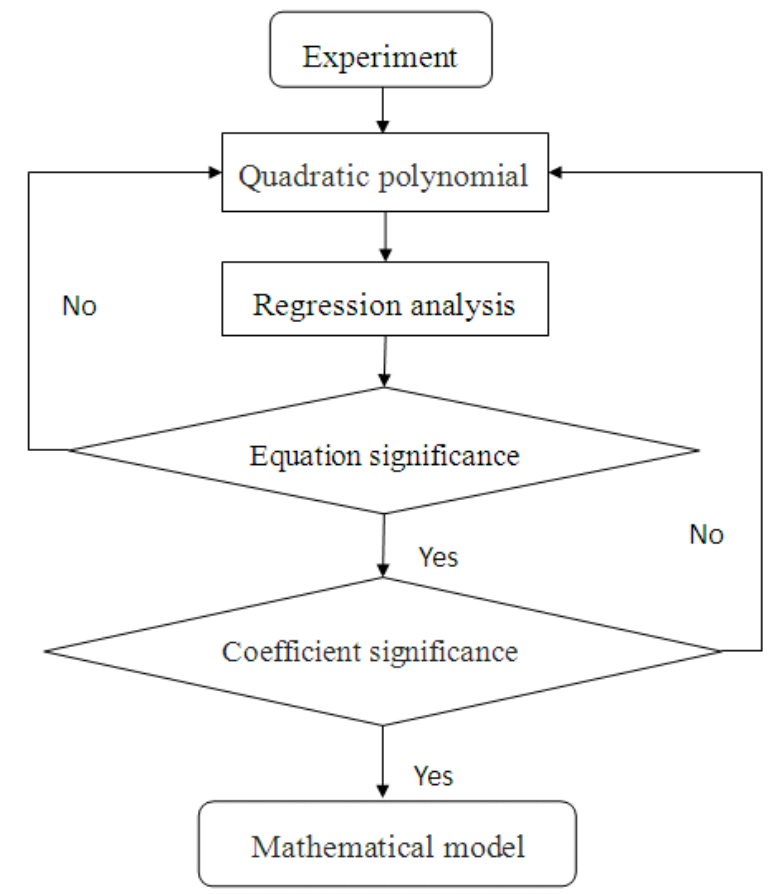

Fig. 3 Block diagram of multiple regression analysis

According to advanced mathematics, the relationship between purifying performance and their factors can be approximated by quadratic polynomial; the multiple regression equation of $\mathrm{P}$ variables can be shown as follows:

$$
\hat{y}=b_{0}+\sum_{j=1}^{p} b_{j} x_{j}+\sum_{i<j} b_{i j} x_{i} x_{j}+\sum_{j=1}^{p} b_{j j} x_{j}^{2}
$$

Where, $\hat{y}$ is objective function.P is variable number. $x_{1}$ is velocity. $x_{2}$ is fiber diameter. $x_{3}$ is areal density.

According to the Figure 3, the mathematical models of the filtration efficiency and filtration resistance were obtained in significant level 0.10 .

$$
\begin{aligned}
& \hat{y}_{1}=5.8070 x_{1}^{2}-1.6137 x_{1}-0.0441 x_{1} x_{3}-0.033 x_{2} x_{3}+320.1 \\
& \hat{y}_{2}=1983.7 x_{1}^{2}-203.4 x_{1}-51.9 x_{2}+1.8 x_{3}-45.3 x_{1} x_{2}-x_{1} x_{3}+311.9
\end{aligned}
$$

Where, $\hat{y}_{1}$ is filtration efficiency, $\hat{y}_{2}$ is filtration resistance.

Comprehensive Evaluation of Purifying Performance.People always except use a function to describe the filte's purifying performance. Therefore, it is important to establish a comprehensive evaluation system for the purifying performance.As to filters, the filtration efficiency are higher and the resistance are lower, the purifying performance are better.Based on the the regression equation above, the unified objective function was established with the direct weighting method as shown in equation 4 . 


$$
f(x)=\sum_{j=1}^{q} \omega_{j} f_{j}(x) \quad j=1,2
$$

Where, $\omega_{j}$ is weighted factor of target function $j$, the value determines the number and importance of the objective function. It is determianed as follows:

If the target function ranges from $\alpha_{j}$ to $\beta_{j}, \omega_{j}=\frac{4}{\left[\alpha_{j}-\beta_{j}\right]^{2}}$, we can get

$$
\omega_{1}=6.44 \times 10^{-4}, \omega_{2}=2.58 \times 10^{-5}
$$

The mathematical model for comprehensive evaluation of purifying performance is as follows:

$$
\begin{aligned}
y= & 5.492 \times 10^{-2} x_{1}^{2}-6.287 \times 10^{-3} x_{1}-1.339 \times 10^{-3} x_{2}+4.644 \times 10^{-5} x_{3}-1.169 \times 10^{-3} x_{1} x_{2} \\
& -5.420 \times 10^{-5} x_{1} x_{3}-2.125 \times 10^{-6} x_{2} x_{3}+0.2141
\end{aligned}
$$

With Equation 5, the comprehensive evaluations on purifying performance of 6 filters are as shown in Table 2.

Tab. 2 Comprehensive evaluation on purifying performance

\begin{tabular}{ccccccc}
\hline Code & $\mathrm{R}_{1}$ & $\mathrm{R}_{2}$ & $\mathrm{Z}_{1}$ & $\mathrm{Z}_{2}$ & $\mathrm{~J}_{1}$ & $\mathrm{~J}_{2}$ \\
Comprehensive evaluation & 0.2056 & 0.2072 & 0.2007 & 0.1863 & 0.2117 & 0.2167 \\
\hline
\end{tabular}

As far as the comprehensive evaluation vaule are concerned, the bigger, the better.As shown in Tab.2, the needle electret filters are better than melt-blown filters, the acupuncture filters are worse. With the change of the surface density and diameter of fibers, the filtering performance of the same kind filter is different. It is found that the purifying performance of filters can be evaluated and compared with Equation 5 availably.

\section{Conclusions}

Comprehensive evaluation system can not only evaluate different filters' purifying performance, but also determine the influence on purifying performance due to different fiber diameter, velocity and areal density. The research can provide important reference to develop, choose and use non-woven filter materials to purify fine particles in ambient air.

\section{Acknowledgements}

This work is supported by Science and Technology Research Project of Hebei Provincial Education Department (ZD2015102) and Key Project of Hebei University of Science and Technology (2013FZ01).

\section{References}

[1] Wei Zhang, Hailan GU. Researches on $\mathrm{PM}_{2.5}$ purifying performance by use of non-woven filtering material[J]. Shanghai Textile Science\&Technology , 2013, 41 ( 2 ) :59-61 (In Chinese)

[2] Wei Zhang, Yiqiang Gao, Xianghong Li. Researches on the Purifying Performance of Filter Materials used in the Comfort Air Conditioning Systems [C].2009:335-248. (In Chinese)

[3] Changsheng Cao, Jianbing Zang, Jiang Cai. Researches on the detection performance of air filters standard.[C].The National Building Materials Testing Laboratory Sustainable Development Forum Proceedings.2014,161-164. (In Chinese)

[4] Tianyi Dai. The Status and Standard of the Test Method of filter material performance in China [J]. Industrial Textiles, 2007, ( 1 ) :31-33. (In Chinese) 
[5] GB/T 14295-2008 Air filters[S].Standardization Administration of the People's Republic of China, 2008(In Chinese)

[6]EN779-2012 Particulate air filters for general ventilation-determination of the filtration performances[S]. Brussels: European Committee for Standardization, 2012.

[7] EN 1822-2009 High efficiency air filters ( HEPA and ULPA ) [S].Brussels: European Committee for Standardization, 2009.

[8] ASHRAE52.2-2007 Method of testing general ventilation air-cleaning devices for removal efficiency by particle size[S].American Society of Heating, Refrigerating and Air-conditioning Engineer, Inc.2007. 